\title{
Function of the N-terminal region of the phosphate- sensing histidine kinase, SphS, in Synechocystis sp. PCC 6803
}

Correspondence

Iwane Suzuki

iwanes6803@biol.tsukuba.ac.jp

Received 19 February 2009

Revised 15 April 2009

Accepted 18 April 2009
Satoshi Kimura, Yoshihiro Shiraiwa and Iwane Suzuki

\author{
Graduate School of Life and Environmental Sciences, University of Tsukuba, Tennodai 1-1-1, \\ Tsukuba 305-8572 Japan
}

\begin{abstract}
In Synechocystis sp. PCC 6803 the histidine kinase SphS (s/l0337) is involved in transcriptional activation of the phosphate $\left(P_{i}\right)$-acquisition system which includes alkaline phosphatase (AP). The $\mathrm{N}$-terminal region of SphS contains both a hydrophobic region and a Per-Arnt-Sim (PAS) domain. The C-terminal region has a highly conserved transmitter domain. Immunological localization studies on heterologously expressed SphS in Escherichia coli indicate that the hydrophobic region is important for membrane localization. In order to evaluate the function of the $\mathrm{N}$-terminal region of SphS, deletion mutants under the control of the native promoter were analysed for in vivo AP activity. Deletion of the $\mathrm{N}$-terminal hydrophobic region resulted in loss of AP activity under both $\mathrm{P}_{\mathrm{i}}$-deficient and $\mathrm{P}_{\mathrm{i}}$-sufficient conditions. Substitution of the hydrophobic region of SphS with that from the $\mathrm{Ni}^{2+}$-sensing histidine kinase, $\mathrm{NrsS}$, resulted in the same induction characteristics as SphS. Deletion of the PAS domain resulted in the constitutive induction of AP activity regardless of $\mathrm{P}_{\mathrm{i}}$ availability. To characterize the PAS domain in more in detail, four amino acid residues conserved in the PAS domain were substituted with Ala. Among the mutants R121A constitutively expressed AP activity, suggesting that R121 is important for the function of the PAS domain. Our observations indicated that the presence of a transmembrane helix in the N-terminal region of SphS is critical for activity and that the PAS domain is involved in perception of $\mathrm{P}_{\mathrm{i}}$ availability.
\end{abstract}

\section{INTRODUCTION}

Phosphorus is an essential element for living organisms and is present in cells predominantly as phosphate. Inorganic phosphate $\left(\mathrm{P}_{\mathrm{i}}\right)$ is incorporated into many cellular components, including nucleotides, nucleic acids, phospholipids and a large variety of metabolic intermediates. It is also important in the covalent modification of proteins via phosphorylation, which is an essential component of many signal transduction and regulatory pathways. Despite its importance phosphorus is one of the least available nutrients in the environment (Schweitzer \& Simon, 1995; Tyrrell, 1999). The responses brought about by $\mathrm{P}_{\mathrm{i}}$ deficiency in Escherichia coli have been extensively studied. A two-component signal transduction system is required for regulation of the expression of the pho regulon

Abbreviations: AP, alkaline phosphatase; PAS, Per-Arnt-Sim; TM, transmembrane.

A supplementary table, listing primers used in this study, and five supplementary figures, showing construction of the modified genes and plasmids used in this study and amino acid alignment of hydrophobic regions from SphS and NrsS, are available with the online version of this paper.
(Makino et al., 1989), which encodes proteins for acquisition, storage and utilization of $\mathrm{P}_{\mathrm{i}}$.

Two-component signal transduction systems consist of two proteins, a histidine kinase and a response regulator (Stock et al., 1989). Typically histidine kinases are membranebound sensory proteins. Once a specific stimulus is perceived by the kinase, a conserved histidine residue in the protein is autophosphorylated and the phosphate group is transferred to a cognate response regulator. The activity of the response regulator is determined by phosphorylation. Since many response regulators affect transcription, this provides a way in which the twocomponent system can modulate physiological responses to intra- and extracellular changes. The regulatory pathway is further complicated by the discovery of a third component modulating the activity of specific twocomponent systems (Chen et al., 2008; Salinas et al., 2007). The $\mathrm{P}_{\mathrm{i}}$-responsive two-component system is present in a wide variety of bacteria. In E. coli it consists of the histidine kinase PhoR, and the response regulator $\mathrm{PhoB}$ (Makino et al., 1989). Disruption of phoB results in complete loss of response to $\mathrm{P}_{\mathrm{i}}$ deficiency, consistent with the two-component system being essential for induction of the pho regulon. A third component, PhoU, is also 
involved in regulation of the pho regulon. Inactivation of phoU constitutively activates expression of the pho regulon via PhoR-PhoB regardless of $\mathrm{P}_{\mathrm{i}}$ availability (Nakata et al., 1984). In E. coli, PhoU thus functions as a negative regulator of the two-component system under $\mathrm{P}_{\mathrm{i}}$-sufficient conditions. Under $\mathrm{P}_{\mathrm{i}}$-deficient conditions the inhibitory effect of PhoU is overcome and the PhoR-PhoB sensory relay activity is restored, resulting in expression of the pho regulon.

In general, histidine kinase proteins consist of two portions: a signal-input domain located at the $\mathrm{N}$ terminus and a transmitter domain located at the $\mathrm{C}$ terminus (Mascher et al., 2006). The former perceives the specific stimulus and modulates the activities of autophosphorylation and phospho-transfer to the cognate response regulator located in the latter domain. Analysis of the Nterminal region in sensory histidine kinases is important in order to understand the mechanism of signal perception. In E. coli, the N-terminal region of PhoR possesses two transmembrane (TM) helices and a Per-Arnt-Sim (PAS) domain (Scholten \& Tommassen, 1993). Deletion of the TM region and/or the PAS domain from PhoR results in constitutive activation of the transmitter domain irrespective of $P_{i}$ availability. This suggests that both domains are involved in repression of kinase activity under $\mathrm{P}_{\mathrm{i}}$-sufficient conditions (Scholten \& Tommassen, 1993; Yamada et al., 1990). In Bacillus subtilis the PhoR protein also contains two TM helices and a PAS domain. However, it has a large periplasmic loop between the TM helices which is absent from PhoR in E. coli. In B. subtilis deletion of the two TM helices and the periplasmic loop from the PhoR protein does not affect induction of the pho regulon, suggesting that the cytosolic transmitter domain senses $P_{i}$ availability and induces expression of the pho regulon (Shi \& Hulett, 1999). The molecular mechanism for sensing the availability of $\mathrm{P}_{\mathrm{i}}$ and regulating it via a histidine kinase thus clearly differs between organisms and the role of specific protein domains warrants further investigation.

Histidine kinases and response regulators are expressed at a very low level and this is likely to be an important factor in downstream gene regulation. Overaccumulation of histidine kinases and response regulators in cells can cause excess signal transduction between cognate partners or between non-cognate partners. (Ehira \& Ohmori, 2006; Krall \& Reed, 2000). This phenomenon has been utilized to screen for novel histidine kinase activities. For example overexpression of the histidine kinase SasA from the cyanobacterium Synechococcus sp. PCC 7942, which functions as a regulator of circadian rhythm, functionally complements mutations of EnvZ and PhoR in E. coli (Nagaya et al., 1993), which function as osmosensors and phosphosensors respectively. Investigation into the specific function of histidine kinases and the role of component domains should thus be conducted under conditions of gene expression equivalent to those of the native histidine kinases in wild-type cells in order to prevent erroneous conclusions. Previous analyses of the signal input domains of PhoR in E. coli and in B. subtilis have utilized multicopy plasmids with strong promoters to express the kinases and it would be advisable to avoid this.

In order to express genetically modified kinases at equivalent levels to the native protein in vivo, the coding sequence of the native gene in the chromosome could be replaced with genes encoding the target proteins via homologous recombination and driven from the original promoter. Since the cyanobacterium Synechocystis sp. PCC 6803 (hereafter referred to as Synechocystis) readily undergoes double homologous recombination between transformed DNA fragments and chromosomal DNA (Williams, 1988), we decided to exploit this ability to study the function of subdomains of the $\mathrm{P}_{\mathrm{i}}$-sensing histidine kinases in this organism utilizing the original promoter. Additionally since most of the genes for histidine kinases in Synechocystis are transcribed in a monocistronic manner there is no need to take the expression levels of the genes downstream from the histidine kinase into account as they are not cotranscribed (Murata \& Suzuki, 2006). These characteristics of Synechocystis greatly simplify studies of genetically modified histidine kinases.

The genome of Synechocystis encodes the histidine kinase SphS (Sll0337) and the response regulator SphR (Slr0081) and a negative regulator SphU (Slr0741), which are structural and functional orthologues of PhoR, PhoB and PhoU respectively (Aiba et al., 1993; Hirani et al., 2001; Juntarajumnong et al., 2007a; Suzuki et al., 2004). In Synechocystis these genes are located on the genome separately and should be transcribed independently. The SphS-SphR pathway regulates the expression of at least 12 genes, including $p h o A$, which encodes a periplasmic alkaline phosphatase (AP) (Suzuki et al., 2004). Induction of AP activity is a highly conserved response to $\mathrm{P}_{\mathrm{i}}$ deficiency in many bacteria and has been used as an indicator of the activity of the SphS-SphR pathway in Synechocystis (Juntarajumnong et al., 2007a). BurutArchanai et al. (2009) have demonstrated that deletion of a portion of the N-terminal sequence of SphS results in constitutive induction of AP activity, indicating that this region is essential to respond to $P_{i}$ fluctuations.

In this report we describe an expression system in Synechocystis cells for modified forms of SphS utilizing the native promoter of the sphS gene, so eliminating any artefacts due to overproduction. We have expressed a series of SphS derivatives and assessed the signal-sensing ability of the modified SphS by measuring cellular AP activity. The possible functions of the hydrophobic region and the PAS-like domain of SphS are discussed.

\section{METHODS}

Bacterial strains and culture conditions. A glucose-tolerant strain of Synechocystis (Williams, 1988) was used as the wild-type strain. The wild-type strain and its derivatives were grown at $34^{\circ} \mathrm{C}$ in BG11 
medium (Stanier et al., 1971) buffered with $20 \mathrm{mM}$ HEPES-NaOH ( $\mathrm{pH}$ 7.5) under continuous illumination provided by incandescent lamps at $70 \mu \mathrm{mol}$ photons $\mathrm{m}^{-2} \mathrm{~s}^{-1}$. Phosphate-free BG11 medium was prepared by substitution of $\mathrm{K}_{2} \mathrm{HPO}_{4}$ with $\mathrm{KCl}$ as described previously (Suzuki et al., 2004). Mutants of SphS were grown in the presence of $25 \mu \mathrm{g}$ kanamycin $\mathrm{ml}^{-1}$ or $25 \mu \mathrm{g}$ spectinomycin $\mathrm{ml}^{-1}$, depending on the inserted antibiotic resistance gene. Liquid cultures were continuously bubbled with $1.0 \%(\mathrm{v} / \mathrm{v}) \mathrm{CO}_{2}$-containing air. $E$. coli strains JM109 (TaKaRa Bio) and BL21(DE3) (Merck KGaA) were used as hosts for genetic manipulation and protein expression respectively, and were grown in LB medium with appropriate antibiotics at $37^{\circ} \mathrm{C}$ (Miller, 1972).

Genetic modifications of the SphS gene in Synechocystis. To replace the entire ORF of the sphS gene with a kanamycin-resistance cassette, DNA fragments upstream and downstream of the ORF of sphS were amplified by PCR with upstream primers, $0337 \mathrm{uF}$ and $0337 \mathrm{uR}$, and downstream primers, $0337 \mathrm{dF}$ and $0337 \mathrm{dR}$, using genomic DNA of Synechocystis as a template. Primer sequences used in this study are shown in Supplementary Table S1, available with the online version of this paper. The upstream fragment was cloned between the NsiI and NdeI sites of pGEM-T easy vector (Promega). This was followed by cloning of the downstream fragment between the SalI and SacII sites of the plasmid containing the upstream fragment to obtain pSK01. All plasmids constructed in this study and their descriptions are shown in Table 1. A kanamycin-resistance cassette, containing NdeI and SalI sites at each end, was amplified by PCR using kanF and kanR primers and EZ-Tn5 (Epicentre) as template. The amplified DNA fragment was digested with NdeI and SalI and inserted into pSK01, which had been cleaved with the same enzymes, to construct pSK02. To obtain the sphS-deleted strain, wildtype cells of Synechocystis were transformed with pSK02 as described by Williams (1988).
In order to replace the kanamycin-resistance cassette with modified sphs genes, a DNA fragment containing the sequence of the transmitter domain of SphS was amplified by PCR using kinF1 and 0337R primers and cloned between the HindIII and SalI sites of pSK02 to obtain pSK03. A spectinomycin-resistance gene cassette, containing SalI sites at both ends, was amplified by PCR using speF and speR primers and the aadA gene in pAM1146 (Tsinoremas et al., 1994) as template. This fragment was cloned into the SalI site of pSK03 to obtain pSK04.

The full-length sphS gene was amplified by PCR using 0337F and 0337R primers to construct the complemented strain (SphSC). The SphS construct lacking a hydrophobic region at the $\mathrm{N}$ terminus ( $s p h S \Delta H y$ ) was obtained by PCR using primers $\Delta$ hyF and 0337R. To construct the PAS-domain-deleted SphS (sphS $\triangle P A S)$, the partial sphS sequences were amplified by PCR using primer pairs 0337F plus PASuR, and PASdF plus 0337R. The two fragments were mixed and further amplified by PCR using $0337 \mathrm{~F}$ and $0337 \mathrm{R}$ primers, generating a $0.95 \mathrm{kbp}$ DNA fragment. The PCR products were cloned into pGEM-T easy vector and the DNA sequences were confirmed. Each of the DNA fragments cloned into pGEM-T easy vector was excised from the vector with $\mathrm{NdeI}$ and HindIII and cloned between the NdeI and HindIII sites of pSK04 to obtain pSK05, pSK06 and pSK07 for strains SphSC, SphS $\Delta \mathrm{Hy}$ and $\mathrm{SphS} \Delta \mathrm{PAS}$, respectively (see Supplementary Fig. S1).

To construct strain SphS $\Delta \mathrm{N}$, expressing the signal-input-domaindeleted SphS, the DNA fragment corresponding to the transmitter domain of SphS was amplified by PCR using kinF2 and 0337R primers. The fragment was cloned between the NdeI and SalI sites of pSK01, and the spectinomycin-resistance cassette was inserted into the plasmid as described above to construct pSK08. $\Delta$ SphS cells were transformed with the plasmids constructed above and spectinomycinresistant cells were selected.

Table 1. Plasmids used in this study

\begin{tabular}{|c|c|c|}
\hline Plasmid & Description & Reference or source \\
\hline pGEM-T easy & Cloning vector, $\mathrm{Ap}^{\mathrm{r}}$ & Promega \\
\hline pAM1146 & Source of $S p^{r}$ cassette, $S p^{r}$ & Tsinoremas et al. (1994) \\
\hline $\mathrm{p} \operatorname{Trc} 99 \mathrm{a}$ & Source of $\mathrm{P}_{t r c}$ and $l a c I^{q}, \mathrm{Ap}^{\mathrm{r}}$ & Amann \& Brosius (1985) \\
\hline pET28a & Expression vector, $\mathrm{Km}^{\mathrm{r}}$ & Merck KGaA \\
\hline pSK01 & $\mathrm{P}_{s p h S}, \mathrm{Ap}^{\mathrm{r}}$ & This study \\
\hline pSK02 & $\mathrm{P}_{s p h S}, s p h S^{-}, \mathrm{Ap}^{\mathrm{r}} \mathrm{Km}^{\mathrm{r}}$ & This study \\
\hline pSK03 & $\mathrm{P}_{\text {sphS }}, \mathrm{Ap}^{\mathrm{r}} \mathrm{Km}^{\mathrm{r}}$ & This study \\
\hline pSK04 & $\mathrm{P}_{s p h S}, \mathrm{Ap}^{\mathrm{r}} \mathrm{Km}^{\mathrm{r}} \mathrm{Sp}^{\mathrm{r}}$ & This study \\
\hline pSK05 & $\mathrm{P}_{s p h s^{-}} s p h S^{+}, \mathrm{Ap}^{\mathrm{r}} S \mathrm{p}^{\mathrm{r}}$ & This study \\
\hline pSK06 & $\mathrm{P}_{\text {sph }}-s p h S \Delta H y^{+}, \mathrm{Ap}^{\mathrm{r}} S \mathrm{p}^{\mathrm{r}}$ & This study \\
\hline pSK07 & $\mathrm{P}_{s p h S^{-}} s p h S \Delta P A S^{+}, \mathrm{Ap}^{\mathrm{r}} S \mathrm{p}^{\mathrm{r}}$ & This study \\
\hline pSK08 & $\mathrm{P}_{s p h S^{-}} \operatorname{sph} S \Delta N^{+}, \mathrm{Ap}^{\mathrm{r}} S \mathrm{p}^{\mathrm{r}}$ & This study \\
\hline pSK09 & $\mathrm{P}_{s p h s^{-}}[n r s S(N)-s p h S \Delta H y]^{+}, \mathrm{Ap}^{\mathrm{r}} S \mathrm{p}^{\mathrm{r}}$ & This study \\
\hline pSK10 & $\mathrm{P}_{s p h s^{-}}[n r s S(N)-s p h S \Delta N]^{+}, \mathrm{Ap}^{\mathrm{r}} S \mathrm{p}^{\mathrm{r}}$ & This study \\
\hline pSK11 & $\mathrm{P}_{\text {sphS }}-\operatorname{sphS}(P 80 A)^{+}, \mathrm{Ap}^{\mathrm{r}} \mathrm{Sp}^{\mathrm{r}}$ & This study \\
\hline pSK12 & $\mathrm{P}_{\text {sphs }}-\operatorname{sphS}(N 96 A)^{+}, \mathrm{Ap}^{\mathrm{r}} \mathrm{Sp}^{\mathrm{r}}$ & This study \\
\hline pSK13 & $\mathrm{P}_{\text {sphs }}-\mathrm{sphS}(R 121 A)^{+}, \mathrm{Ap}^{\mathrm{r}} \mathrm{Sp}^{\mathrm{r}}$ & This study \\
\hline pSK14 & $\mathrm{P}_{\text {sphs }}-\mathrm{sphS}(E 124 A)^{+}, \mathrm{Ap}^{\mathrm{r}} \mathrm{Sp}^{\mathrm{r}}$ & This study \\
\hline pSK15 & $\mathrm{P}_{\text {sphs }}-\mathrm{sph} S(H 207 A)^{+}, \mathrm{Ap}^{\mathrm{r}} \mathrm{Sp}^{\mathrm{r}}$ & This study \\
\hline pSK16 & $\mathrm{P}_{t r c}-\operatorname{sphS}(H 207 A)^{+}, \mathrm{Ap}^{\mathrm{r}} \mathrm{Sp}^{\mathrm{r}}$ & This study \\
\hline pSK17 & $\mathrm{P}_{t r c}-s p h S \Delta H y(H 207 A)^{+}, \mathrm{Ap}^{\mathrm{r}} \mathrm{Sp}^{\mathrm{r}}$ & This study \\
\hline pSK18 & $\mathrm{P}_{t r c}-[n r s S(N)-s p h S(H 207 A) \Delta H y]^{+}, \mathrm{Ap}^{\mathrm{r}} \mathrm{Sp}^{\mathrm{r}}$ & This study \\
\hline
\end{tabular}


Construction of fusion-protein-expressing strain. To express a fusion protein of the N-terminal part of the nickel-sensing histidine kinase from Synechocystis, NrsS (Lopez-Maury et al., 2002), and SphS, a DNA fragment corresponding to the $\mathrm{N}$-terminal region (amino acid residues 1-222) of $n r s S$ was amplified by PCR using primer pair nrsSF and nrsSR. The resultant fragment was cloned into the NdeI site of pSK06 or pSK08 to construct pSK09 and pSK10 (see Supplementary Fig. S2). The direction of the inserted fragment was confirmed by PCR using nrsSF and 0337R primers. $\Delta$ SphS cells were transformed with pSK09 or pSK10 to yield strains expressing $\mathrm{NrsS}(\mathrm{N})-\mathrm{SphS} \Delta \mathrm{Hy}$ and $\operatorname{NrsS}(\mathrm{N})-\mathrm{SphS} \Delta \mathrm{N}$, respectively.

Construction of strains expressing point-mutated SphS. In order to substitute the conserved amino acid residues in the PAS domain of SphS with Ala, the sphS gene containing the targeted substitution was synthesized by a fusion PCR strategy (Wang et al., 2002). This resulted in point-mutated sphS fragments (see Supplementary Fig. S3). The fragments were then digested with $\mathrm{NdeI}$ and $\mathrm{XhoI}$ and cloned between the NdeI and XhoI sites of pSK04. $\Delta$ SphS cells were transformed with the plasmids, resulting in strains expressing SphS(P80A), SphS(N96A), SphS(R121A), SphS(E124A), and $\operatorname{SphS}(\mathrm{H} 207 \mathrm{~A})$.

Measurement of AP activity. Wild-type and mutant strains of Synechocystis grown in BG11 medium for $16 \mathrm{~h}$ were harvested by centrifugation at $25{ }^{\circ} \mathrm{C}$ at $3000 \mathrm{~g}$ for $5 \mathrm{~min}$ and the cells were washed twice with phosphate-free BG11 medium. The cells were subsequently inoculated into BG11 or phosphate-free BG11 medium at $\mathrm{OD}_{730} 0.2$ 0.4 and further incubated for $24 \mathrm{~h}$. The activity of AP in the intact cells was measured by the rate of degradation of $p$-nitrophenyl phosphate (Aiba et al., 1993).

Preparation of recombinant SphS protein and raising an antibody. The coding region of the sphS gene in pSK05 was cleaved by NdeI and SalI and cloned into pET28a (Merck KGaA) digested with both enzymes. The resulting plasmid, pET-SphS, was then introduced into E. coli BL21(DE3) cells (Supplementary Fig. S3).

E. coli transformants were grown at $37^{\circ} \mathrm{C}$ in LB medium supplemented with $25 \mu \mathrm{g}$ kanamycin $\mathrm{ml}^{-1}$. The recombinant protein was induced in exponentially growing cells by $1 \mathrm{mM}$ IPTG. After $4 \mathrm{~h}$ incubation, cells were harvested by centrifugation, washed with buffer A [50 mM HEPES/NaOH (pH 7.5), $100 \mathrm{mM} \mathrm{NaCl}$ ], and suspended in buffer $\mathrm{A}$. The cells were then disrupted by sonication at $4{ }^{\circ} \mathrm{C}$ using a UD-201 sonicator (TOMY). The cell extract was centrifuged at $20000 \mathrm{~g}$ for $10 \mathrm{~min}$ and the inclusion body containing recombinant protein was collected. This was washed twice with buffer B [50 mM HEPES/NaOH (pH 7.5), $100 \mathrm{mM} \mathrm{NaCl}, 5 \mathrm{mM}$ EDTA, $0.05 \%$ (w/v) sodium deoxycholate, $0.5 \mathrm{mg}$ lysozyme $\mathrm{ml}^{-1}$ ] at room temperature for $30 \mathrm{~min}$. The inclusion body was suspended in buffer C [50 $\mathrm{mM}$ HEPES/NaOH (pH 7.5), $100 \mathrm{mM} \mathrm{NaCl}, 8 \mathrm{M}$ urea]. Insoluble material was removed by centrifugation and the supernatant containing His-tagged SphS was loaded onto a HiTrap chelating column (GE Healthcare Bio-Science) and washed with buffer C. Bound proteins were eluted with buffer C containing $250 \mathrm{mM}$ imidazole.

Eluted proteins were subjected to $12.5 \%$ SDS-PAGE and the gels were stained as described by Ortiz et al. (1992). The band corresponding to the recombinant protein was excised and the gel slice crushed and suspended in electrophoresis buffer (Laemmli, 1970). Protein was eluted from the gel by shaking for $12 \mathrm{~h}$ at room temperature, filtered with a $5 \mu \mathrm{m}$ centrifugal filter device (Ultrafree-MC, Millipore) and the purified recombinant SphS was used to immunize a rabbit to obtain an antibody against SphS.

Localization of SphS in E. coli. To express SphS, SphS $\Delta$ Hy and $\mathrm{NrsS}(\mathrm{N})-\mathrm{SphS} \Delta \mathrm{Hy}$ in E. coli, we constructed pSK16, 17 and 18 which include the entire sequence for SphS or modified SphS substituting the phosphorylatable His residue with Ala under control of the strong artificial trc promoter (see Supplementary Fig. S4). Mutation of the His residues in SphS derivatives was included to inactivate the kinase activity, so minimizing possible effects of overproduction of the kinases on the growth of E. coli cells. To exchange the H207 of $\mathrm{SphS} \Delta \mathrm{Hy}$ with alanine, the DNA fragment between the $\mathrm{XbaI}$ and $\mathrm{XhoI}$ sites of pSK06 was exchanged with that of pSK15, producing plasmid pSK06a. In order to introduce the trc promoter into pSK15 and pSK06a, a DNA fragment including the trc promoter was amplified by PCR using trcF and trcR primers and pTrc99a (Amann \& Brosius, $1985)$ as template. After digesting the amplified DNA fragment with $\mathrm{NdeI}$, it was cloned into the NdeI site of pSK15 and pSK06a; the resulting plasmids were named pSK16 and pSK17, respectively. The direction of the inserted trc promoter was confirmed by PCR. To construct plasmid pSK18 the trc promoter sequence was amplified by PCR using trcF2 and trcR primers and pTrc99a DNA as the template. The amplified DNA was digested with NsiI and NdeI, and inserted between the NsiI and NdeI sites of pSK17. The amplified nrsS DNA fragment as described above was digested with $N d e I$ and introduced into the NdeI site of the resulting plasmid.

Plasmids pSK16, pSK17 and pSK18, and a control plasmid, pSK05, were introduced into E. coli JM109 and the transformants were cultured in LB medium at $37^{\circ} \mathrm{C}$. Cells in the exponential growth phase were collected by centrifugation, resuspended in two different buffers systems: buffer D [50 mM Tris/ $\mathrm{HCl}(\mathrm{pH} 8.0), 50 \mathrm{mM} \mathrm{NaCl}]$ or buffer E [buffer D containing $100 \mathrm{mM} \mathrm{Na}_{2} \mathrm{CO}_{3}$ (pH 11.5)] at $4{ }^{\circ} \mathrm{C}$ and disrupted by sonication. Clear cell lysates obtained after removal of cell debris by centrifugation at $20000 \mathrm{~g}$ for $10 \mathrm{~min}$ were then ultracentrifuged at $160000 \mathrm{~g}$, for $1 \mathrm{~h}$ at $4{ }^{\circ} \mathrm{C}$. The supernatant was withdrawn and termed the cytosolic protein fraction. The precipitates were washed by suspending in the same buffer used for sonication and recovering by ultracentrifugation under the same conditions. In order to distinguish between membrane-bound proteins and insoluble matter which was not removed by the initial centrifugation, the final pellet was resuspended in buffer $F[50 \mathrm{mM}$ Tris/ $\mathrm{HCl}$ ( $\mathrm{pH} 8.0), 50 \mathrm{mM} \mathrm{NaCl}, 1 \%(\mathrm{v} / \mathrm{v})$ Tween 20] and insoluble matter was removed by centrifugation. The detergent-soluble fraction was designated the membrane proteins.

For Western blot analysis, proteins separated by SDS-PAGE were electro-transferred to PVDF membrane. Rabbit anti-SphS serum and alkaline-phosphatase-conjugated anti-rabbit IgG (Bio-Rad) were used as the primary and secondary antibodies. The primary antibody was used at a $1: 1,000$ dilution with TBS [Tris-buffered saline; $20 \mathrm{mM}$ Tris/HCl ( $\mathrm{pH} 7.5$ ), $0.5 \mathrm{M} \mathrm{NaCl}$ ] containing $1 \%$ (w/v) skim milk and the secondary antibody was used at a $1: 20000$ with TBS. Detection was carried out using the amplified Alkaline Phosphatase GAR kit according to manufacturer's instructions (Bio-Rad). A sample (10 $\mu \mathrm{g}$ protein) from each fraction was subjected to $10 \%$ SDS-PAGE. Protein concentrations were determined by the Bio-Rad Protein Assay kit, with BSA as standard.

\section{RESULTS AND DISCUSSION}

\section{Construction of domain-deleted SphS expressing strains}

Juntarajumnong et al. (2007b) have reported that the translation start site of the sphS gene is located at the ATG $141 \mathrm{bp}$ upstream from the GTG originally assigned as the translation initiation codon (Kaneko et al., 1996, http:// bacteria.kazusa.or.jp/cyanobase/). The newly annotated SphS consists of 430 amino acid residues and contains a 
hydrophobic stretch in the N-terminal region, a transmitter domain in the C-terminal region, and a putative PAS domain between them (Fig. 1). This is a structure which many orthologues of SphS have. To investigate molecular mechanisms of signal perception by SphS, we decided to express the domain-deleted SphS from the original promoter of the sphS gene and examine its responsiveness in vivo using the AP reporter protein activity. This strategy ensures that the sensor molecule is maintained at the same level as in the wild-type. To express genetically modified SphS in cells of Synechocystis from the native promoter of the sphS gene, the entire ORF of the sphS was substituted with a kanamycin-resistance gene cassette (Fig. 1a). Segregation of the sphS-deleted chromosome was confirmed by PCR (data not shown) and the strain, named $\Delta$ SphS, was utilized as a host for secondary transformations introducing modified SphS. The kanamycin-resistance gene of $\Delta S$ phS was replaced with the modified sphS genes containing a spectinomycin-resistance gene cassette which was inserted immediately downstream of the termination codon of sphS. (Supplementary Fig. S1). The strains SphS $\Delta \mathrm{N}$, SphS $\Delta$ Hy and SphS $\Delta$ PAS contained deletions in the entire $\mathrm{N}$-terminal region (amino acid residues 2-196) excluding the transmitter domain, the hydrophobic region (amino acid residues 2-50), and the putative PAS domain (amino acid residues 69-177) respectively. The intact sphS gene was also introduced to obtain a complemented strain, SphSC. Replacement of all the $\Delta$ SphS chromosomal copies with the inserted genes was confirmed by PCR and sensitivity to kanamycin (data not shown). All the spectinomycin-resistant strains grew well in $\mathrm{P}_{\mathrm{i}}$-sufficient conditions (data not shown).

\section{Effects of the domain deletions of SphS on the expression of AP activity}

Since the expression of the gene for AP is solely regulated by the SphS-SphR pathway in Synechocystis (Suzuki et al., 2004), we assayed AP activity to evaluate functionality of the SphS derivatives (Fig. 1b). The AP activity in cells of $\Delta$ SphS was negligible under both $\mathrm{P}_{\mathrm{i}}$-sufficient and -deficient conditions. AP activity in response to $\mathrm{P}_{\mathrm{i}}$ availability in the SphSC strain was similar to that observed in the wild-type. This indicates that the SphSC protein was functioning normally regardless of the base substitutions introduced into the upstream and the downstream coding regions to insert the gene constructs into NdeI and SalI sites; these substitutions did not alter the amino acid sequence. Similarly, insertion of a spectinomycin-resistance gene cassette downstream of the coding region did not cause any alterations to the normal function of the gene. We next analysed the AP activities in response to $\mathrm{P}_{\mathrm{i}}$ availability in cells of the domain-deleted strains. SphS $\Delta \mathrm{N}$ and SphS $\Delta \mathrm{Hy}$ did not exhibit any AP activity at any level of $\mathrm{P}_{\mathrm{i}}$ availability. This suggests that the hydrophobic domain in the $\mathrm{N}$-terminal region of SphS is essential for sensing $\mathrm{P}_{\mathrm{i}}$ availability and/or activating kinase activity in vivo. Cells of SphS $\triangle$ PAS constitutively expressed AP activity regardless of $\mathrm{P}_{\mathrm{i}}$ availability, as has been reported for cells of the sphUdeleted strain (Juntarajumnong et al., 2007a), suggesting that the PAS domain might function to suppress SphS activity under $\mathrm{P}_{\mathrm{i}}$-sufficient conditions, possibly together with SphU. The construct containing the hydrophobic domain minus the PAS domain (SphS $\triangle \mathrm{PAS}$ ) exhibited constitutive kinase activity. However, the construct minus the hydrophobic domain but containing the PAS domain
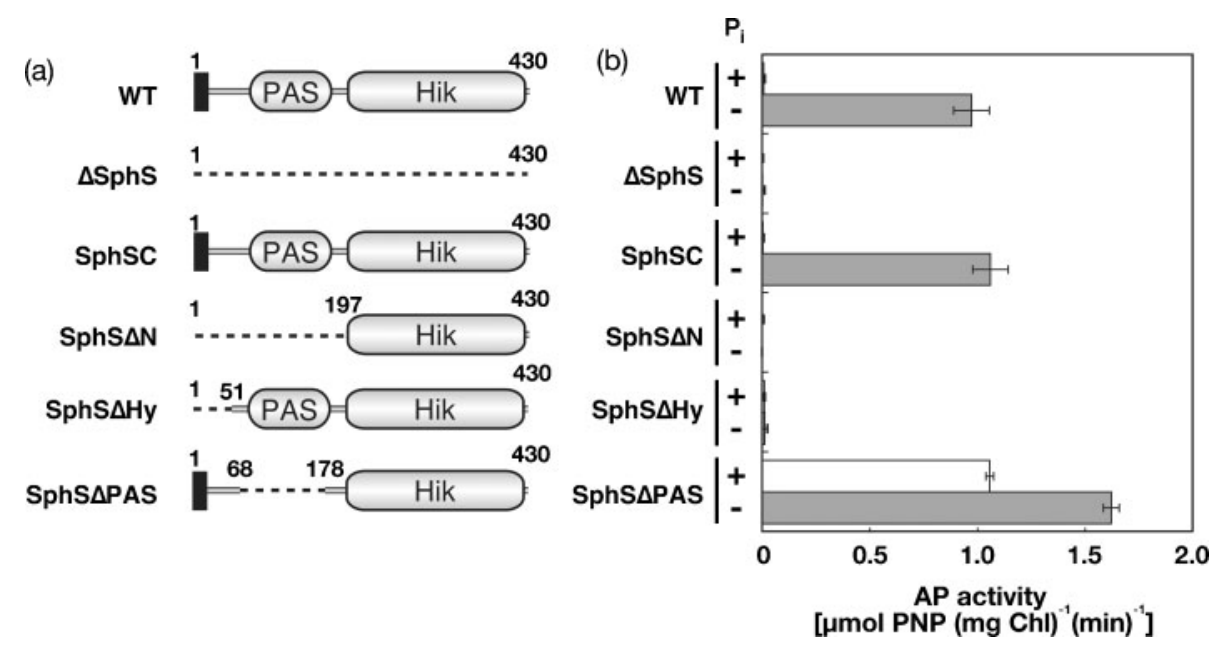

Fig. 1. Effects of deletion of domains in SphS on the expression of AP activity. (a) Domain architectures of native and mutated SphS. The domains were depicted with the Pfam program (Finn et al., 2008). Numbers indicate the positions of amino acid residues from the translation start site of the native SphS. Dashed lines represent the regions deleted from the native protein. PAS, Hik and black rectangles indicate PAS domain, transmitter domain of the histidine kinase and the hydrophobic region, respectively. (b) AP activities in each strain grown under $P_{i}$-sufficient $\left(P_{i}+\right.$, white bars $)$ or $P_{i}$-deficient $\left(P_{i}-\right.$, shaded bars $)$ conditions for $24 \mathrm{~h}$. The assays were carried out three times with cultures grown independently; means \pm SEM are plotted. 
(SphS $\Delta \mathrm{Hy}$ ) was inactive. This indicates that the hydrophobic domain is essential for activation of the transmitter domain.

\section{Signal perception/kinase activation only requires a hydrophobic region and is not sequence- specific}

To investigate the dependence of the specific amino acid sequence of the SphS hydrophobic domain on kinase activation and sensing we decided to completely replace this region with the amino acid sequence from the $\mathrm{Ni}^{2+}$. sensing histidine kinase NrsS (Lopez-Maury et al., 2002). These two hydrophobic regions have very little amino acid sequence similarity (see Supplementary Fig. S5). We constructed strains that expressed a fusion protein with the N-terminal region of NrsS and the truncated forms of SphS to obtain $\operatorname{NrsS}(\mathrm{N})-\mathrm{SphS} \Delta \mathrm{N}$ and $\mathrm{NrsS}(\mathrm{N})-\mathrm{SphS} \Delta \mathrm{Hy}$ (Fig. 2a). NrsS upregulates the $n r s B A C D$ operon coding for an $\mathrm{ABC}$ transporter involved in $\mathrm{Ni}^{2+}$ tolerance in the presence of $\mathrm{Ni}^{2+}$ in Synechocystis. NrsS has two membrane-spanning helices at the $\mathrm{N}$-terminal region and is predicted to be located in the plasma membrane (LopezMaury et al., 2002). If the hydrophobic domain of SphS is essential in sensing changes in $\mathrm{P}_{i}$ availability, replacement with the hydrophobic region from NrsS may result in loss of $\mathrm{P}_{\mathrm{i}}$ sensing.

AP activity in cells expressing $\mathrm{NrsS}(\mathrm{N})-\mathrm{SphS} \Delta \mathrm{Hy}$ responded to $\mathrm{P}_{\mathrm{i}}$ availability to the same extent as those expressing SphSC. However, whilst the SphSC-expressing strain did not have any AP activity under $\mathrm{P}_{\mathrm{i}}$-sufficient conditions, detectable activity was present in the $\mathrm{NrsS}(\mathrm{N})$ SphS $\Delta$ Hy-expressing strain. We also looked at AP activity in the cells of the strain expressing $\mathrm{NrsS}(\mathrm{N})-\mathrm{SphS} \Delta \mathrm{N}$, which has the PAS domain deleted, and as for SphS $\triangle$ PAS, the activity was constitutive (Figs 1 and 2).
These findings suggest that the hydrophobic region of SphS might not be essential for signal perception and/or kinase activation. It also indicates that the presence of any hydrophobic region may be sufficient for induction of AP activity by SphS.

\section{The $\mathbf{N}$-terminal region of SphS is essential for membrane localization}

To investigate the region of SphS that may be required for membrane localization, and hence probably signal perception, we designed a series of constructs which were truncated/deleted in specific domains. We also generated an antibody that was capable of recognizing all of the domains in the protein. Following expression and fractionation into membrane and soluble fractions it should be possible to determine the location of the protein immunologically.

The antibody could specifically detect the recombinant SphS via Western blotting at a level of $5 \mathrm{ng}$ (data not shown). At first, we attempted to detect SphS protein in wild-type cells. Cells in the exponential growth phase were collected and disrupted by sonication. After removing the unbroken cells and cell debris, the cell lysate was separated into cytoplasmic, plasma membrane and thylakoid membrane fractions by discontinuous sucrose-density-gradient centrifugation (Murata \& Omata, 1988). When $10 \mu \mathrm{g}$ of each sample was subjected to Western blotting analysis, no protein was detected (data not shown). This indicates that the amount of SphS in vivo is below the detection limit; this is perhaps not surprising, as sensor proteins are liable to be of very low abundance.

We next attempted to overexpress SphS in Synechocystis. The $\Delta$ SphS strain was transformed with plasmid pSK16 or pSK17, which contain the sphS and sphS $\Delta H y$ gene, (a)

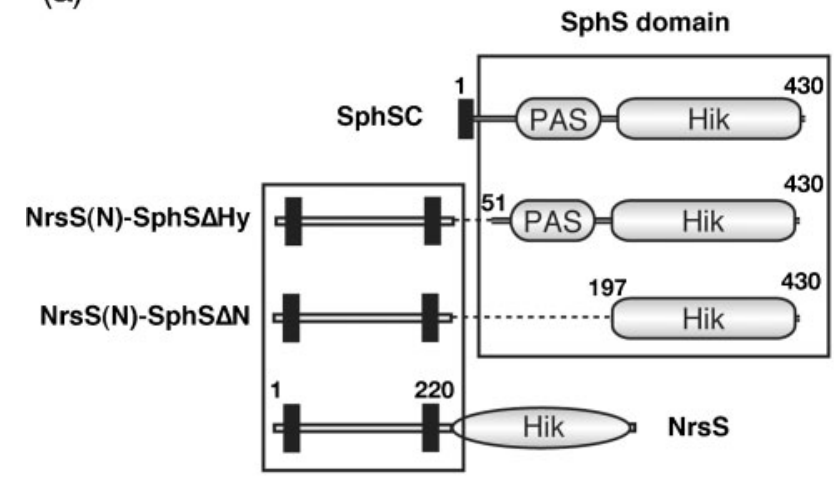

NrsS domain $[\mathrm{NrsS}(\mathrm{N})]$

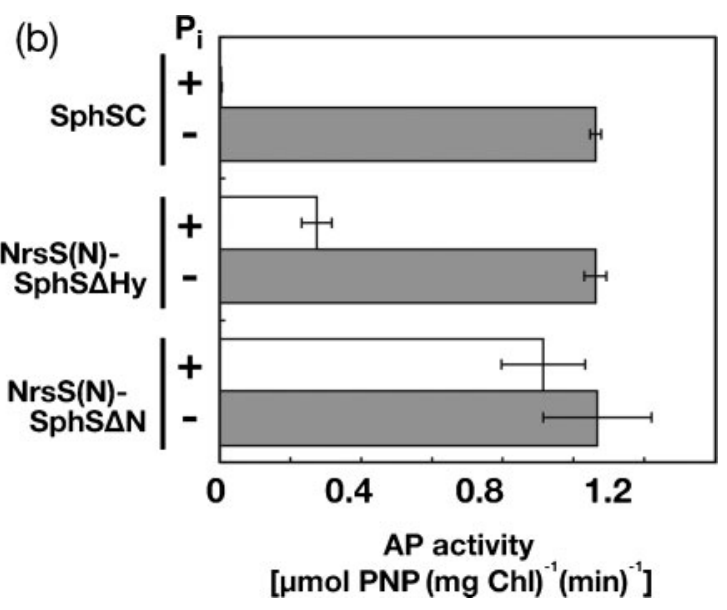

Fig. 2. Fusions of truncated SphS constructs with the TM region of NrsS. (a) Domain architectures of the fusion proteins; (b) AP activities under $P_{i}$-sufficient $\left(P_{i}+\right.$, white bars) or $P_{i}$-deficient $\left(P_{i}-\right.$, shaded bars $)$ conditions. The assays were carried out three times with cultures grown independently; means \pm SEM are plotted. 
respectively, under the control of $\mathrm{P}_{\text {tro }}$ a strong bacterial artificial promoter (Supplementary Fig. S4). To regulate expression levels of the introduced gene, the $\operatorname{lacl}^{q}$ gene was concomitantly integrated into the chromosome via homologous recombination (Supplementary Fig. S4). To minimize unexpected effects of the overexpression of the sensor proteins, the phosphorylatable His residues of both proteins were substituted with an Ala residue. After transformation, spectinomycin-resistant colonies of both transformants were obtained. However, we could not cultivate the cells in liquid BG11 medium containing $25 \mu \mathrm{g}$ spectinomycin ml ${ }^{-1}$ even without addition of IPTG. The increased level of SphS in these cells may be causing deleterious effects on cellular function. The precise mechanism for this is unclear.

Since elevated expression of SphS, and its derivatives, in Synechocystis was deleterious we decided to investigate expression in a heterologous system. SphS, SphS $\Delta \mathrm{Hy}$ and $\mathrm{NrsS}(\mathrm{N})-\mathrm{SphS} \Delta \mathrm{Hy}$ were successfully expressed in $E$. coli cells and fractionated for immunological determination of subcellular localization. We used two different $\mathrm{pH}$ treatments for preparation of the soluble and membrane fractions, $\mathrm{pH} 8.0$ and $\mathrm{pH}$ 11.5. The latter $\mathrm{pH}$ was employed to overcome any problems which might be associated with proteins being trapped inside membrane vesicles. Treatment of membranes at $\mathrm{pH} 11.5$ is a welldocumented method of linearizing closed membrane vesicles (Griff et al., 1992). If a positive result was obtained on $\mathrm{pH} 8.0$ isolated membranes and a negative one with $\mathrm{pH} 11.5$ membranes this would indicate that the result was not due to membrane insertion but rather to trapping of the protein inside a closed vesicle. True membrane insertion could only be verified if the protein was detected in both $\mathrm{pH} 8.0$ and $\mathrm{pH} 11.5$ preparations. All the membrane preparations isolated at $\mathrm{pH} 8.0$ gave a positive signal (Fig. 3). SphS $\Delta \mathrm{Hy}$ could be solubilized after the $\mathrm{pH} 11.5$ treatment, indicating that it is not membrane inserted. SphS and $\mathrm{NrsS}(\mathrm{N})-\mathrm{SphS} \Delta \mathrm{Hy}$, which contain the hydrophobic domain, remained membrane bound after $\mathrm{pH} 11.5$ treatment, consistent with the hydrophobic domain being essential for membrane localization.

\section{Identification of critical amino acid residues in the PAS domain}

Our results above indicate that the PAS domain may be involved in the regulation of kinase activity depending on $P_{i}$ availability (Figs 1, 2, and 4). To identify amino acid residues required for PAS domain function, we aligned PAS domains from several orthologues of SphS, including PhoR from E. coli (Fig. 4a) and identified four absolutely conserved hydrophilic residues that could be critically important. It is noteworthy that the PAS domains of cyanobacterial SphS proteins have lower identity with that of PhoR, but the function is still conserved. Thus the conserved amino acid residues might have an important role for the function of the domain. The four conserved residues P80, N96, R121 and E124, and autophosphorylatable His residue, H207, in SphS, were separately substituted with $\mathrm{Ala}$ and introduced into $\Delta \mathrm{SphS}$ Synechocystis cells. Point mutations of P80A and E124A did not influence the induction of AP activity, suggesting that these residues are not critical for activity of the PAS domain in SphS (Fig. 4b). Mutations of N96A and H207A resulted in loss of induction of AP activity regardless of $\mathrm{P}_{\mathrm{i}}$ availability. N96 might be important for the activation of the transmitter domain since substitution resulted in complete inactivation. Mutation of R121A caused constitutive expression of the AP activity even under $\mathrm{P}_{\mathrm{i}}{ }^{-}$ sufficient conditions, suggesting that R121 may have an important function in sensing by the PAS domain.

In E. coli, PhoR lacking its TM region constitutively expresses the AP gene, suggesting that this region is required for negative regulation of PhoR activity. In SphS of Synechocystis, deletion of part of the hydrophobic region (amino acid residues 8-15) also constitutively induces AP (a)
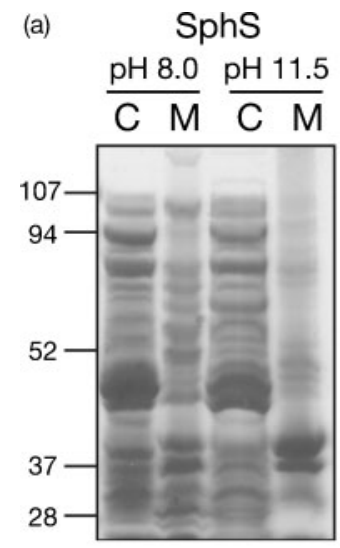

(b)

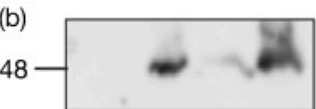

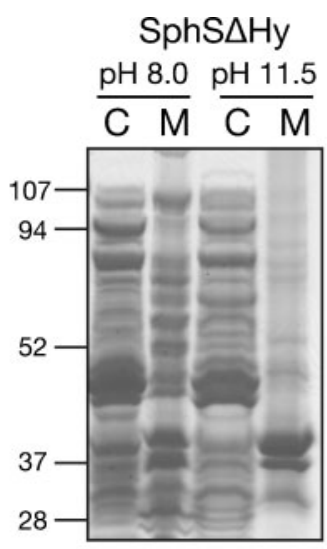

$43-1-$
$\mathrm{NrsS}(\mathrm{N})-\mathrm{SphS} \Delta \mathrm{Hy}$ $\mathrm{pH} 8.0 \mathrm{pH} 11.5$ $\overline{\mathrm{C} M} \overline{\mathrm{CM}}$
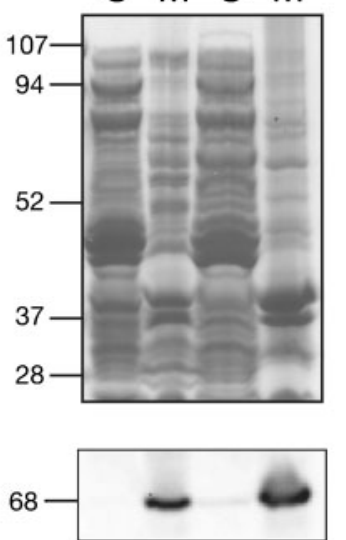

Fig. 3. Western blot analysis of SphS and modified SphS proteins expressed in cells of $E$. coli. Cells of $E$. coli expressing the native and modified SphS proteins were disrupted at $\mathrm{pH} 8.0$ and at $\mathrm{pH} 11.5$. The crude extracts were further separated by ultracentrifugation into cytosolic (C) and membrane (M) fractions. A $10 \mu \mathrm{g}$ sample of each fraction was subjected to SDS-PAGE. Gels were stained with Coomassie brilliant blue (a) or electroblotted on PVDF membranes and detected with an anti-SphS polyclonal antibody (b). These results were reproducible; representative results are presented. Numbers indicate molecular mass (kDa). 
(a)

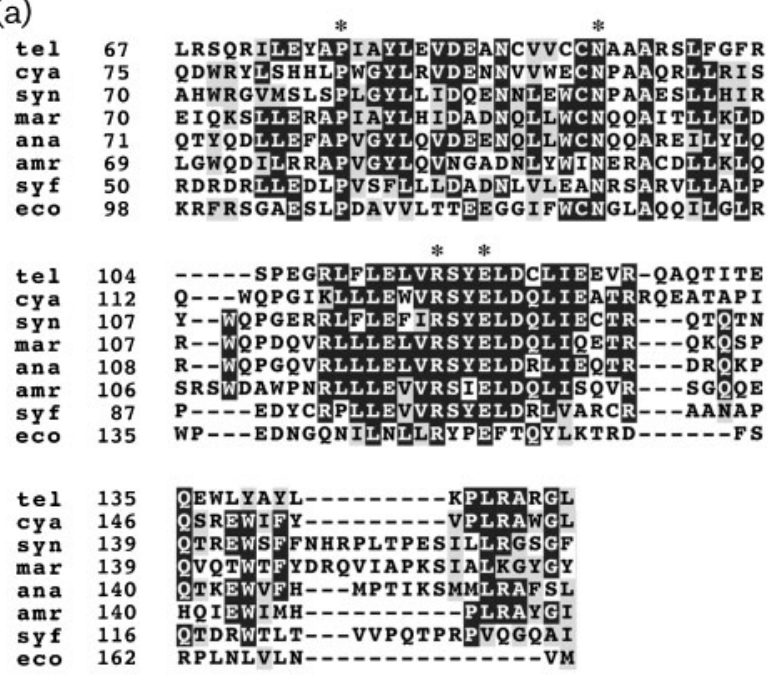

(b)

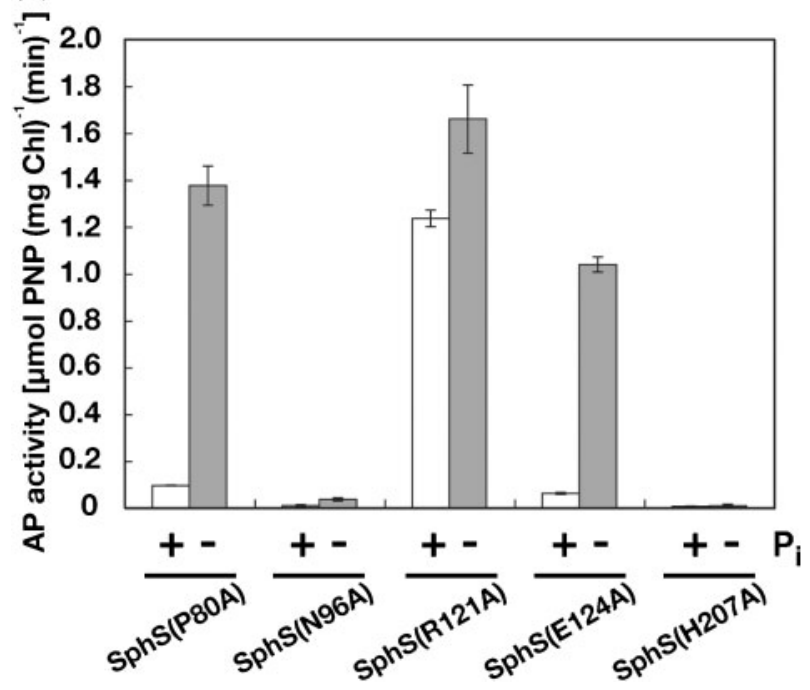

Fig. 4. Effects of point mutations in the PAS domain on the expression of AP activity. (a) Alignment of amino acid sequences of the PAS domain of SphS orthologues. tel, Thermosynechococcus elongatus BP-1 (NP 681715); cya, Synechococcus sp. JA-3-3Ab (YP 475742); syn, Synechocystis sp. PCC 6803 (NP_442021); mar, Microcystis aeruginosa NIES-843 (YP_001660279); ana, Anabaena sp. PCC 7120 (NP_488542); amr, Acaryochloris marina MBIC11017 (YP_001519542); syf, Synechococcus elongatus PCC 7942 (YP_400028); eco, Escherichia coli K-12 MG1655 (NP_414934). Asterisks indicate the amino acid residues substituted with Ala. Numbers indicate positions of amino acid residues. The amino acid alignment was created by CLUSTAL W (Thompson et al., 1994). (b) AP activity in the cells grown under $\mathrm{P}_{\mathrm{i}}$-sufficient $\left(\mathrm{P}_{\mathrm{i}}+\right.$, white bars $)$ or $\mathrm{P}_{\mathrm{i}}$-deficient $\left(\mathrm{P}_{\mathrm{i}}-\right.$, grey bars $)$ conditions. The assays were carried out three times with cultures grown independently; means \pm SEM are plotted.

expression (Burut-Archanai et al., 2009). Our results indicate that deletion of the entire hydrophobic region of SphS was unable to activate reporter AP activity regardless of
$\mathrm{P}_{\mathrm{i}}$ availability. Moreover, a protein in which the hydrophobic region of SphS was replaced by that from NrsS retained $\mathrm{P}_{\mathrm{i}}$ responsiveness, suggesting that the hydrophobic region does not play a role in sensing the $\mathrm{P}_{\mathrm{i}}$ signal but rather is important in localizing the sensory protein to a specific location on the membrane. There are several examples of histidine kinases in bacteria being located at the poles of cells (Hallez et al., 2007). The hydrophobic region of SphS might be important to localize it to a specific location. In order to detect SphS expressed in vivo in Synechocystis new techniques will have to be developed.

In E. coli, it has been suggested that the activity of PhoR is repressed through formation of a hetero complex with PhoU and the high-affinity $P_{i}$ transporter (Wanner, 1993). No direct evidence of such protein interaction has been demonstrated (Baek et al., 2007). In this study we found that recombinant SphS expressed in E. coli was localized to the membrane via the hydrophobic domain. This suggested that a similar situation may occur in Synechocystis but we were not able to demonstrate it because of the low amount of SphS in this cyanobacterium. The presence of this domain is required for activation of kinase activity. The repressive function of the PAS domain under $\mathrm{P}_{\mathrm{i}}$-sufficient conditions was similar to that of the SphU protein. It may be that the SphU protein interacts with SphS via the PAS domain. This could be tested by looking for direct proteinprotein interactions. The mutants which we have generated affecting the function of the PAS domain, such as R121A and N96A, could be very useful materials to demonstrate the interaction between SphS and SphU genetically.

\section{ACKNOWLEDGEMENTS}

We are grateful to Professor A. R. Slabas and Dr J. Rowland (University of Durham, UK) for their constructive discussion. This work was partially supported by a Grant-in-Aid for Scientific Research on Priority Areas (17051032).

\section{REFERENCES}

Aiba, H., Nagaya, M. \& Mizuno, T. (1993). Sensor and regulator proteins from the cyanobacterium Synechococcus species PCC7942 that belong to the bacterial signal-transduction protein families: implication in the adaptive response to phosphate limitation. Mol Microbiol 8, 81-91.

Amann, E. \& Brosius, J. (1985). 'ATG vectors' for regulated high-level expression of cloned genes in Escherichia coli. Gene 40, 183-190.

Baek, J. H., Kang, Y. J. \& Lee, S. Y. (2007). Transcript and protein level analyses of the interactions among PhoB, PhoR, PhoU and CreC in response to phosphate starvation in Escherichia coli. FEMS Microbiol Lett 277, 254-259.

Burut-Archanai, S., Incharoensakdi, A. \& Eaton-Rye, J. J. (2009). The extended N-terminal region of SphS is required for detection of external phosphate levels in Synechocystis sp. PCC 6803. Biochem Biophys Res Commun 378, 383-388.

Chen, E. J., Sabio, E. A. \& Long, S. R. (2008). The periplasmic regulator ExoR inhibits ExoS/ChvI two-component signalling in Sinorhizobium meliloti. Mol Microbiol 69, 1290-1303. 
Ehira, S. \& Ohmori, M. (2006). NrrA directly regulates expression of het $R$ during heterocyst differentiation in the cyanobacterium Anabaena sp. strain PCC 7120. J Bacteriol 188, 8520-8525.

Finn, R. D., Tate, J., Mistry, J., Coggill, P. C., Sammut, S. J., Hotz, H. R., Ceric, G., Forslund, K., Eddy, S. R. \& other authors (2008). The Pfam protein families database. Nucleic Acids Res 36, D281-D288.

Griff, I. C., Schekman, R., Rothman, J. E. \& Kaiser, C. A. (1992). The yeast SEC17 gene product is functionally equivalent to mammalian $\alpha$ SNAP protein. J Biol Chem 267, 12106-12115.

Hallez, R., Mignolet, J., van Mullem, V., Wery, M., Vandenhaute, J., Letesson, J. J., Jacobs-Wagner, C. \& De Bolle, X. (2007). The asymmetric distribution of the essential histidine kinase PdhS indicates a differentiation event in Brucella abortus. EMBO J 26, 1444-1455.

Hirani, T. A., Suzuki, I., Murata, N., Hayashi, H. \& Eaton-Rye, J. J. (2001). Characterization of a two-component signal transduction system involved in the induction of alkaline phosphatase under phosphate-limiting conditions in Synechocystis sp. PCC 6803. Plant Mol Biol 45, 133-144.

Juntarajumnong, W., Hirani, T. A., Simpson, J. M., Incharoensakdi, A. \& Eaton-Rye, J. J. (2007a). Phosphate sensing in Synechocystis sp. PCC 6803: SphU and the SphS-SphR two-component regulatory system. Arch Microbiol 188, 389-402.

Juntarajumnong, W., Incharoensakdi, A. \& Eaton-Rye, J. J. (2007b). Identification of the start codon for sphS encoding the phosphatesensing histidine kinase in Synechocystis sp. PCC 6803. Curr Microbiol 55, 142-146.

Kaneko, T., Sato, S., Kotani, H., Tanaka, A., Asamizu, E., Nakamura, Y., Miyajima, N., Hirosawa, M., Sugiura, M. \& other authors (1996). Sequence analysis of the genome of the unicellular cyanobacterium Synechocystis sp. strain PCC6803. II. Sequence determination of the entire genome and assignment of potential protein-coding regions. DNA Res 3, 109-136.

Krall, L. \& Reed, J. W. (2000). The histidine kinase-related domain participates in phytochrome B function but is dispensable. Proc Natl Acad Sci U S A 97, 8169-8174.

Laemmli, U. K. (1970). Cleavage of structural proteins during the assembly of the head of bacteriophage T4. Nature 227, 680-685.

Lopez-Maury, L., Garcia-Dominguez, M., Florencio, F. J. \& Reyes, J. C. (2002). A two-component signal transduction system involved in nickel sensing in the cyanobacterium Synechocystis sp. PCC 6803. Mol Microbiol 43, 247-256.

Makino, K., Shinagawa, H., Amemura, M., Kawamoto, T., Yamada, M. \& Nakata, A. (1989). Signal transduction in the phosphate regulon of Escherichia coli involves phosphotransfer between PhoR and PhoB proteins. J Mol Biol 210, 551-559.

Mascher, T., Helmann, J. D. \& Unden, G. (2006). Stimulus perception in bacterial signal-transducing histidine kinases. Microbiol Mol Biol Rev 70, 910-938.

Miller, J. H. (1972). Experiments in Molecular Genetics. Cold Spring Harbor, NY: Cold Spring Harbor Laboratory.

Murata, N. \& Omata, T. (1988). Isolation of cyanobacterial plasma membrane. Methods Enzymol 167, 245-250.

Murata, N. \& Suzuki, I. (2006). Exploitation of genomic sequences in a systematic analysis to access how cyanobacteria sense environmental stress. J Exp Bot 57, 235-247.

Nagaya, M., Aiba, H. \& Mizuno, T. (1993). Cloning of a sensorykinase-encoding gene that belongs to the two-component regulatory family from the cyanobacterium Synechococcus sp. PCC7942. Gene 131, 119-124.

Nakata, A., Amemura, M. \& Shinagawa, H. (1984). Regulation of the phosphate regulon in Escherichia coli K-12: regulation of the negative regulatory gene $p h o U$ and identification of the gene product. J Bacteriol 159, 979-985.

Ortiz, M. L., Calero, M., Fernandez Patron, C., Patron, C. F., Castellanos, L. \& Mendez, E. (1992). Imidazole-SDS-Zn reverse staining of proteins in gels containing or not SDS and microsequence of individual unmodified electroblotted proteins. FEBS Lett 296, 300304.

Salinas, P., Ruiz, D., Cantos, R., Lopez-Redondo, M. L., Marina, A. \& Contreras, A. (2007). The regulatory factor SipA provides a link between NblS and NblR signal transduction pathways in the cyanobacterium Synechococcus sp. PCC 7942. Mol Microbiol 66, 1607-1619.

Scholten, M. \& Tommassen, J. (1993). Topology of the PhoR protein of Escherichia coli and functional analysis of internal deletion mutants. Mol Microbiol 8, 269-275.

Schweitzer, B. \& Simon, M. (1995). Growth limitation of planktonic bacteria in a large mesotrophic lake. Microb Ecol 30, 89-104.

Shi, L. \& Hulett, F. M. (1999). The cytoplasmic kinase domain of PhoR is sufficient for the low phosphate-inducible expression of pho regulon genes in Bacillus subtilis. Mol Microbiol 31, 211-222.

Stanier, R. Y., Kunisawa, R., Mandel, M. \& Cohen-Bazire, G. (1971). Purification and properties of unicellular blue-green algae (order Chroococcales). Bacteriol Rev 35, 171-205.

Stock, J. B., Ninfa, A. J. \& Stock, A. M. (1989). Protein-phosphorylation and regulation of adaptive responses in bacteria. Microbiol Rev 53, 450-490.

Suzuki, S., Ferjani, A., Suzuki, I. \& Murata, N. (2004). The SphS-SphR two component system is the exclusive sensor for the induction of gene expression in response to phosphate limitation in Synechocystis. J Biol Chem 279, 13234-13240.

Thompson, J. D., Higgins, D. G. \& Gibson, T. J. (1994). CLUSTAL W: improving the sensitivity of progressive multiple sequence alignment through sequence weighting, position-specific gap penalties and weight matrix choice. Nucleic Acids Res 22, 4673-4680.

Tsinoremas, N. F., Kutach, A. K., Strayer, C. A. \& Golden, S. S. (1994). Efficient gene transfer in Synechococcus sp. strains PCC 7942 and PCC 6301 by interspecies conjugation and chromosomal recombination. J Bacteriol 176, 6764-6768.

Tyrrell, T. (1999). The relative influences of nitrogen and phosphorus on oceanic primary production. Nature 400, 525-531.

Wang, H. L., Postier, B. L. \& Burnap, R. L. (2002). Polymerase chain reaction-based mutageneses identify key transporters belonging to multigene families involved in $\mathrm{Na}^{+}$and $\mathrm{pH}$ homeostasis of Synechocystis sp. PCC 6803. Mol Microbiol 44, 1493-1506.

Wanner, B. L. (1993). Gene regulation by phosphate in enteric bacteria. J Cell Biochem 51, 47-54.

Williams, J. G. K. (1988). Construction of specific mutations in photosystem II photosynthetic reaction center by genetic engineering methods in Synechocystis 6803. Methods Enzymol 167, 766-778.

Yamada, M., Makino, K., Shinagawa, H. \& Nakata, A. (1990). Regulation of the phosphate regulon of Escherichia coli: properties of phoR deletion mutants and subcellular localization of PhoR protein. Mol Gen Genet 220, 366-372.

Edited by: C.-C. Zhang 